\title{
TFE3 regulates renal adenocarcinoma cell proliferation via activation of the $m$ TOR pathway
}

\author{
YUAN FANG ${ }^{1,2}$, WEI BAO ${ }^{2}$, QIU RAO ${ }^{2}$, XUAN WANG $^{2}$,

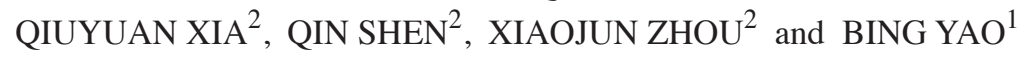 \\ ${ }^{1}$ Reproductive Medical Center and ${ }^{2}$ Department of Pathology, Jinling Hospital, Clinical Medical \\ School of Southern Medical University, Nanjing, Jiangsu 210002, P.R. China
}

Received August 17, 2016; Accepted May 15, 2017

DOI: $10.3892 / \mathrm{mmr} .2017 .6930$

\begin{abstract}
The present study aimed to investigate the role of transcription factor E3 (TFE3) in the regulation of proliferation in renal adenocarcinoma cells. The LV-TFE3 overexpression (OE) lentivirus and negative control CON195 (NC) lentivirus were transfected into the ACHN cell line. Protein expression of FLAG-tag TFE3 was determined using western blot analysis. Differences in cell proliferation, plate clone formation and cell cycle distribution between $\mathrm{OE}$ and $\mathrm{NC}$ groups were compared using MTT, plate colony formation and flow cytometry assays, respectively. The levels of mammalian target of rapamycin (mTOR) and phosphorylated ribosomal protein S6 (p-rpS6) were analyzed by western blotting. Cell proliferation and colony formation increased significantly in the $\mathrm{OE}$ group compared with the $\mathrm{NC}$ group. The \% of cells in the G1 and G2 phases of the cell cycle decreased, while the $\%$ of cells in the $\mathrm{S}$ phase of the cell cycle increased in the OE group compared with the NC group. In addition, mTOR and p-rpS6 levels were increased in the OE group compared with the NC group. The results of the present study demonstrated that TFE3 overexpression resulted in increased ACHN cell proliferation and plate clone formation. TFE3 may promote renal tumor growth by regulating cell cycle progression and activating the phosphatidylinositol 3-kinase/AKT serine/threonine kinase 1/mTOR signaling pathway.
\end{abstract}

Correspondence to: Professor Bing Yao, Reproductive Medical Center, Jinling Hospital, Clinical Medical School of Southern Medical University, 305 East Zhongshan Road, Nanjing, Jiangsu 210002, P.R. China

E-mail: yaobing@nju.edu.cn

Professor Xiaojun Zhou, Department of Pathology, Jinling Hospital, Clinical Medical School of Southern Medical University, 305 East Zhongshan Road, Nanjing, Jiangsu 210002, P.R. China

E-mail: zhouxj3456@126.com

Key words: renal adenocarcinoma, transcription factor E3, proliferation, cell cycle, mammalian target of rapamycin, ribosomal protein S6

\section{Introduction}

Xp11.2 translocation renal cell carcinomas (RCCs) are the most common subtype of pediatric RCCs, in addition to affecting $\sim 1.5 \%$ of adults $(1,2)$. Xp11.2 translocation RCCs were first identified to be a novel, genetically distinct disease in the 2010 World Health Organization renal tumor classification (3). Xp11.2 translocation RCCs are characterized by chromosomal translocations involving the Xp11.2 breakpoint, resulting in transcription factor E3 (TFE3) gene fusion. TFE3 fusion with other regulatory elements and TFE3 overexpression is a notable feature of Xp11.2 translocation RCCs (4).

TFE3 is a member of the basichelix-loop-helix leucine zipper family of transcription factors. TFE3 interacts with transcriptional regulators, including E2F transcription factor 3 (E2F3), SMAD family member 3 and lymphoid enhancer-binding factor 1 , and serves an important role in cell growth and cell proliferation (1). In addition, translocations/TFE3 fusions have been directly implicated in tumorigenesis (5). However, the association between growth-related factors and the cell cycle-related factors that regulate the signal transduction pathways of TFE3-overexpressing cells has not been investigated.

Although Xp11.2 translocation RCCs predominantly occur in children and adolescents, the prognosis for adult translocation RCC is particularly poor (6). The TFE3-mediated direct transcriptional upregulation of the Met tyrosine kinase receptor triggers the activation of downstream signaling pathways, such as the phosphatidylinositol 3-kinase (PI3K)/AKT serine/threonine kinase 1 (AKT)/mammalian target of rapamycin (mTOR) pathway and the Ras/mitogen-activated protein kinase (MAPK) pathway (7). Temsirolimus directly interferes with the PI3K/AKT/mTOR signaling pathway by acting on mTOR and decreasing the activity of effector molecules, including phosphorylated ribosomal protein S6 (p-rpS6). Parikh et al (8) reported the successful treatment of adult Xp11.2 translocation RCC via temsirolimus. However, Choueiri et al (9) reported that certain patients with advanced translocation RCC develop progressive disease following temsirolimus treatment. Therefore, the role of mTOR in Xp11.2 translocation RCCs requires further investigation.

In the present study, renal adenocarcinoma $\mathrm{ACHN}$ cells were infected with the lentivirus LV-TFE3 to produce a stable TFE3-overexpressing cell line. Subsequently, the effects of 
TFE3 overexpression on cell proliferation, plate clone formation, cell cycle distribution and the activation of the mTOR signaling pathway were examined.

\section{Materials and methods}

Cell culture. Human embryonic kidney 293T cells (293T) and human renal adenocarcinoma ACHN cells were purchased from the Cell Bank of Type Culture Collection of the Chinese Academy of Sciences (Shanghai, China). Cells were cultured in Dulbecco's modified Eagle's medium (Gibco; Thermo Fisher Scientific, Inc., Waltham, MA, USA) supplemented with $10 \%$ fetal bovine serum (Gibco; Thermo Fisher Scientific, Inc.). The cell lines were cultured at $37^{\circ} \mathrm{C}$ with $5 \% \mathrm{CO}_{2}$ and saturated humidity. When the cells grew to $75 \%$ confluence, they were harvested for further analysis.

Human TFE3 overexpression (OE) lentivirus package. The packaging GV341 plasmid (Shanghai GeneChem Co., Ltd., Shanghai, China) was transfected into $293 \mathrm{~T}$ cells using Lipofectamine 2000 (Invitrogen; Thermo Fisher Scientific, Inc.), according to the manufacturer's protocol. The cells were placed onto complete medium and cultured for $48 \mathrm{~h}$ prior to the supernatant being collected, and the titer was detected via titration analysis. The human TFE3 gene (National Center for Biotechnology Information GenBank accession no. NM_006521) fused with a FLAG-tag was synthesized by Shanghai GeneChem Co., Ltd. (Shanghai, China), to construct the LV-TFE3 OE vector. The empty vector was used for the negative control (NC) group. The lentiviral vector also contained the green fluorescent protein (GFP) gene. The lentivirus titer was $2 \times 10^{8}$ transducing units $(\mathrm{TU}) / \mathrm{ml}$ in the $\mathrm{OE}$ group and $1 \times 10^{9} \mathrm{TU} / \mathrm{ml}$ in the $\mathrm{NC}$ group. ACHN cells were seeded at a concentration of $4 \times 10^{4}$ cells/well in 6-well culture plates, cultivated in the incubator for $12 \mathrm{~h}$, and subsequently infected with $\mathrm{OE}$ or $\mathrm{NC}$ lentivirus at a multiplicity of infection of 10. The two lentiviruses in the $\mathrm{OE}$ and $\mathrm{NC}$ groups contained a puromycin-resistant cassette, which confers puromycin resistance to eukaryotic cells. The puromycin resistance gene is routinely used as a selectable marker for stably-transformed mammalian cell lines. The infected cells were cultivated for a further $72 \mathrm{~h}$, and $2 \mu \mathrm{g} / \mathrm{ml}$ puromycin was added to each well. Following $48 \mathrm{~h}$ in culture, an inverted fluorescence microscope was used to analyze efficiency of overexpression (magnification, $\mathrm{x} 100)$.

Western blot analysis. Total protein was isolated from cells using radioimmunoprecipitation assay lysis buffer (Sigma-Aldrich; Merck KGaA, Darmstadt, Germany). A bicinchoninic acid assay was used to measure the total protein concentration. Equal amounts of protein $(20 \mu \mathrm{g})$ were separated by $10 \%$ SDS-PAGE and subsequently transferred onto a polyvinylidene fluoride membrane (EMD Millipore, Billerica, MA, USA). The membranes were blocked in TBS-Tween 20 with $5 \%$ skim milk at room temperature for $1 \mathrm{~h}$. The membranes were incubated with murine antibody against FLAG-tag (1:3,000; cat. no. F1804; Sigma-Aldrich; Merck KGaA), rabbit polyclonal antibody against mTOR (1:3,000; cat. no. 2936-1; Epitomics; Abcam, Cambridge, UK), rabbit polyclonal antibody against p-rpS6 (1:1,000; cat. no. 4858; Cell Signaling Technology, Inc., Danvers, MA, USA) and murine antibody against human GADPH $(1: 2,000$; cat. no. SC-32233; Santa Cruz Biotechnology, Inc., Dallas, TX, USA) at $4^{\circ} \mathrm{C}$ overnight. The membranes were subsequently incubated with appropriate peroxidase-conjugated secondary antibody mouse immunoglobulin G $(1: 5,000$; cat. no. sc-2005; Santa Cruz Biotechnology, Inc.). Antibody binding was visualized using enhanced chemiluminescence solution (Pierce; Thermo Fisher Scientific, Inc.). GADPH was used as an endogenous control.

MTT assay. The cells were seeded into 96-well plates (2,000 cells/well) and incubated for $24 \mathrm{~h}$, and subsequently treated with $20 \mu 15 \mathrm{~g} / 1$ MTT (Gen-View Scientific, Inc., El Monte, FL, USA) and incubated at $37^{\circ} \mathrm{C}$ for $4 \mathrm{~h}$. The supernatants were removed and $150 \mu 1$ dimethyl sulfoxide (Sigma-Aldrich; Merck KGaA) was added to each well. The absorbance at $450 \mathrm{~nm}$ was measured using a microplate reader. The optical density value was measured each day for 5 days, and growth curves were produced.

Plate colony formation assay. Cells were seeded in 6-well plates; each group had three replicate wells and contained 500 cells/well. After 14 days, the cell colonies were visible to the naked eye. The cells were stained with Giemsa (cat. no. AR-0752; Shanghai Dingguo Biotech Co., Ltd., Shanghai, China) and the number of visible colonies containing $>50$ cells were quantified.

Analysis of cell cycle distribution using flow cytometry. Following 5 days of transduction, the cells were trypsinized, washed with PBS, and fixed in $70 \%$ ethanol at $4^{\circ} \mathrm{C}$ overnight. Cell cycle distribution analysis was performed using a cell cycle detection kit (BD Biosciences, Franklin Lakes, NJ, USA). The cells were incubated with propidium iodide at $37^{\circ} \mathrm{C}$ for $30 \mathrm{~min}$ in the dark. The cell cycle was analyzed using BD FACSArray ${ }^{\mathrm{TM}}$ software (v6.0) on a BD FACSVerse flow cytometer (BD Biosciences).

Statistical analysis. Each experiment was repeated at least three times to ensure experimental repeatability. Data were expressed as the mean \pm standard error of the mean, and analyses were performed using SPSS software (version 19.0; IBM Corp., Armonk, NY, USA). One-way analysis of variance followed by the Tukey test was used to compare multiple groups. $\mathrm{P}<0.05$ was considered to indicate a statistically significant difference.

\section{Results}

Overexpression of TEF3 in ACHN cells. ACHN cells in the OE (TFE3-overexpressing lentivirus) were cultured to $90 \%$ confluence and GFP fluorescence was observed under an inverted fluorescence microscope (Fig. 1A-D). Following puromycin selection, the majority of the cells in the $\mathrm{OE}$ group were positive for GFP fluorescence, suggesting a stable overexpressing cell line was successfully constructed by the lentivirus transduction. The protein expression levels of TFE3 were also detected using western blot analysis (Fig. 1E), and TFE3 overexpression in the OE cells was confirmed. 
A

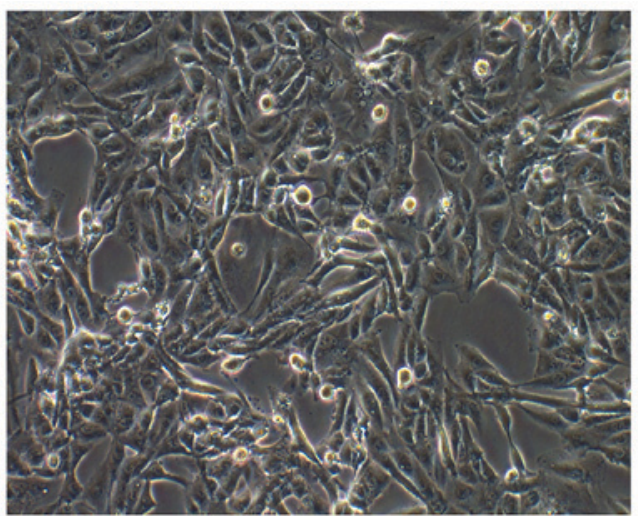

C
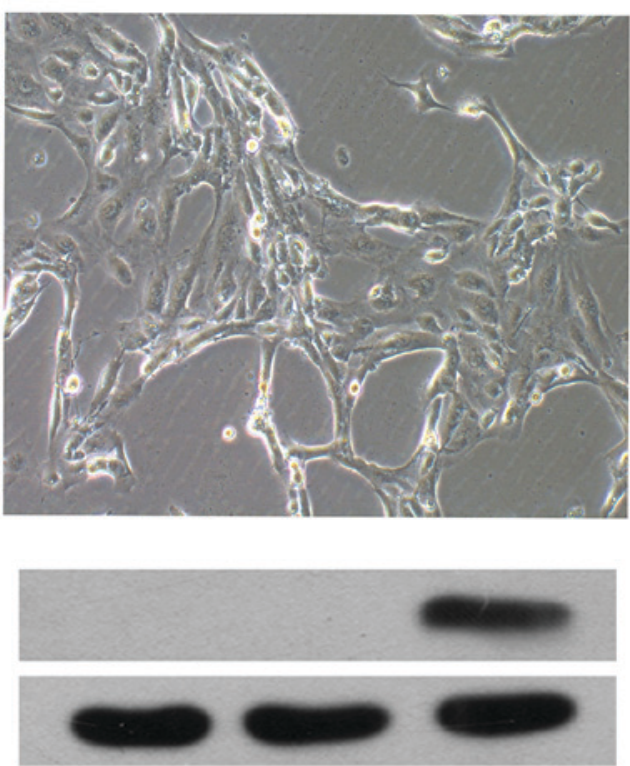

Con
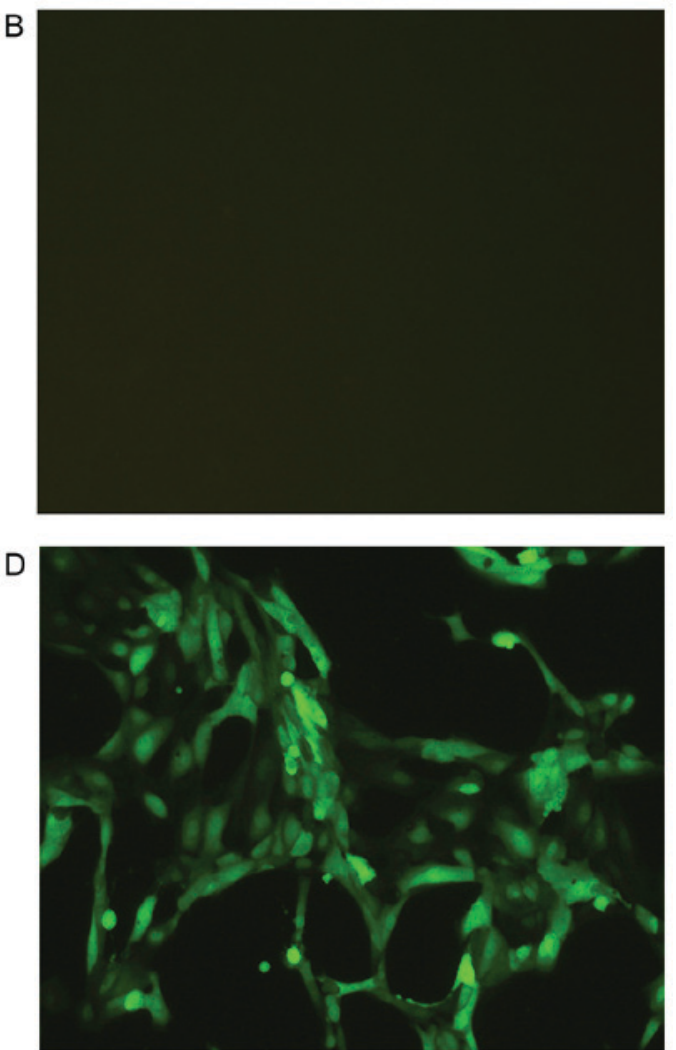

Flag-tag (95 kDa)

GAPDH $(36 \mathrm{kDa})$

Figure 1. TFE3 overexpression in ACHN cells. Visualization of ACHN cells in the OE group (A and B) prior to puromycin selection, and (C and D) following $48 \mathrm{~h}$ of puromycin selection. Magnification, x100. (E) Western blot analysis of TFE3 expression in the three experimental groups. FLAG-tagged TFE3 was detected at $95 \mathrm{kDa}$. TFE3, transcription factor E3; OE, overexpression; NC, negative control; Con, control.

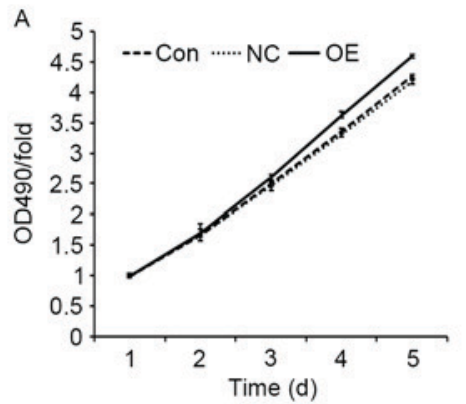

B

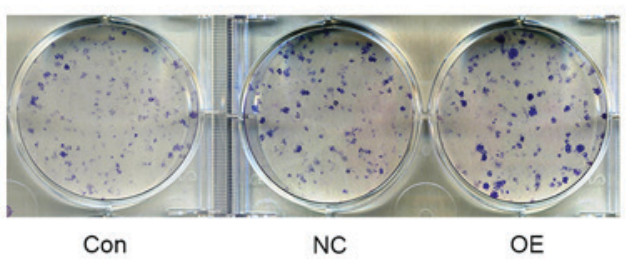

C

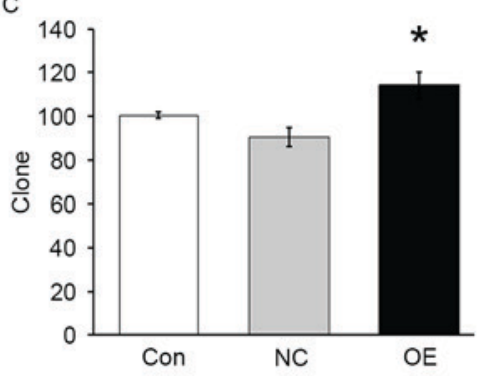

Figure 2. TFE3 overexpression promotes ACHN cell growth. (A) Results of the MTT assay. (B) Colony formation assay. (C) Quantitative analysis of colonies in the Con, $\mathrm{NC}$ and $\mathrm{OE}$ groups. The data are expressed as the mean \pm standard error. " $\mathrm{P}<0.05$ vs. NC group. TFE3, transcription factor E3; OE, overexpression; $\mathrm{NC}$, negative control; Con, control; OD, optical density.

TFE3 overexpression enhances ACHN cell growth and proliferation. In order to evaluate the effect of TFE3 overexpression on the proliferation of ACHN cells, an MTT assay was performed. The results demonstrated that, under the same growth conditions, the growth rate was increased in the OE group compared with the $\mathrm{NC}$ group $(\mathrm{P}<0.05$; Fig. $2 \mathrm{~A})$, indicating that overexpression of TFE3 promoted the growth of ACHN cells. In addition, the colony formation assay demonstrated that the number of colonies in the NC and OE groups were $90 \pm 5$ and $114 \pm 6$, respectively. The colony formation ability was significantly increased in the $\mathrm{OE}$ group compared with the NC group ( $\mathrm{P}<0.05$; Fig. $2 \mathrm{~B}$ and $\mathrm{C})$. 


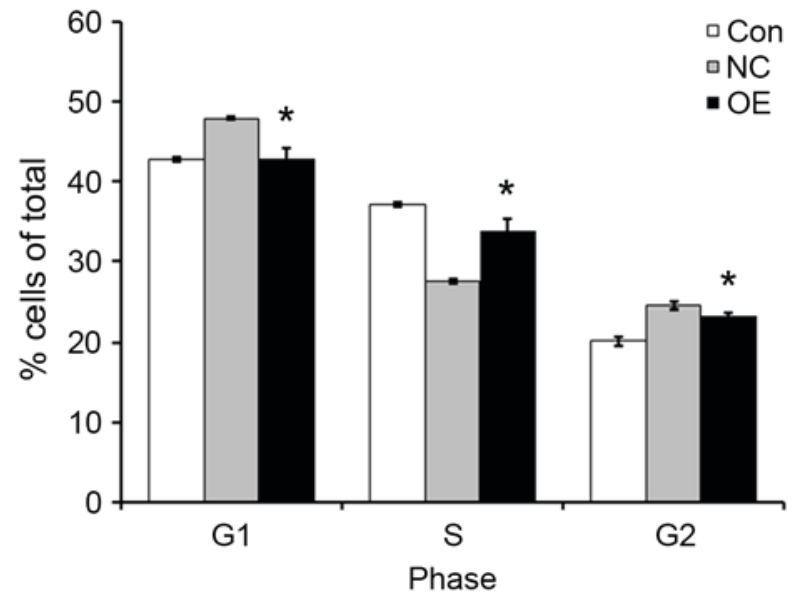

Figure 3. TFE3 overexpression promotes ACHN cell cycle progression. The $\%$ of cells in different phases of the cell cycle were analysed by flow cytometry in the three experimental groups. The data are expressed as the mean \pm standard error of the mean. ${ }^{*} \mathrm{P}<0.05$ vs. NC group. TFE3, transcription factor $\mathrm{E} 3$; OE, overexpression; $\mathrm{NC}$, negative control; Con, control.

TFE3 overexpression promotes the cell cycle progression of ACHN cells. In order to further investigate how TFE3 overexpression enhanced the proliferation of ACHN cells, cell cycle profiles were examined by flow cytometry. Compared with the $\mathrm{NC}$ group, the OE group exhibited decreased percent of cells in the G1 and G2 phases of the cell cycle, and increased percent of cells in the S phase (Fig. 3). The results of the present study indicated that TEF3 promoted ACHN cell cycle progression.

TFE3 overexpression activates the mTOR pathway in ACHN cells. mTOR and p-rpS6 protein expression levels were detected using western blot analysis. Compared with the NC group, mTOR and p-rpS6 levels were upregulated in the OE group (Fig. 4). The results of the present study suggested that TFE3 may be involved in activating the mTOR pathway in ACHN cells.

\section{Discussion}

Xp11.2 translocation RCCs have been increasingly demonstrated to be a subset of RCC characterized by a variety of chromosomal translocations involving the TFE3 gene with a breakpoint at Xp11.2, resulting in fusion with a number of translocation partners, including papillary renal cell carcinoma, ASPSCR1 UBX domain containing tether for SLC2A4, splicing factor proline and glutamine rich, clathrin heavy chain and non-POU domain containing octamer-binding (10). TFE3 protein is constitutively overexpressed in Xp11.2 translocation RCCs $(4,11)$. Rao et al (12) suggested that TFE3-positive pediatric RCCs may be associated with poorer outcomes and higher stages (III/IV), compared with TFE3-negative RCCs. In addition, patients with Xp11.2 translocation RCCs frequently present at an advanced stage and demonstrate a more invasive clinical course and poorer prognosis, compared with patients with non-Xp11.2 translocation RCCs (13). However, the cytological mechanisms by which TFE3 overexpression may regulate the proliferation and cell cycle distribution in a renal adenocarcinoma model have not been reported.

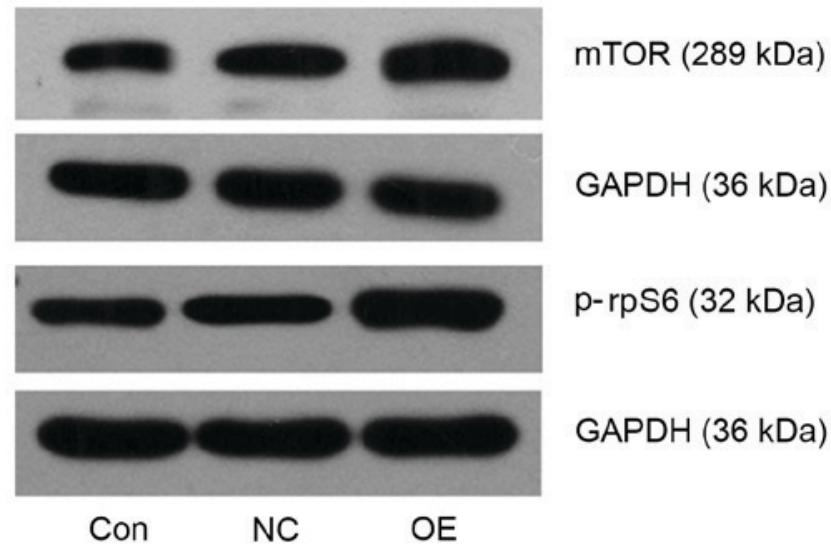

Figure 4. TFE3 overexpression activates mTOR pathway activation in ACHN cells. Western blot analysis demonstrated increased mTOR and p-rpS6 expression in the OE group compared with the NC and Con groups. GAPDH was used as a loading control. TFE3, transcription factor E3; mTOR, mammalian target of rapamycin; p-rpS6, phosphorylated ribosomal protein $\mathrm{S} 6$; OE, overexpression; NC, negative control; Con, control.

In the present study, the TFE3 gene sequence was introduced in the human renal adenocarcinoma ACHN cells via a recombinant lentivirus. Puromycin was used to select and enrich for the infected cells and the fluorescence emitted from the GFP marker was observed under an inverted fluorescence microscope. The present study established TFE3-overexpressing cells and observed, through growth curves, that the TFE3-overexpressing cells grew more rapidly compared with the negative control group. In addition, the plate colony-forming assays demonstrated that TFE3 overexpression resulted in increased colony formation capacity compared with the negative control group. The results of the present study indicated that TFE3 overexpression promoted ACHN cell proliferation. Ploper et al (14) reviewed the role of micropthalmia-associated transcription factor (MITF) in lysosomal biogenesis, and how cancers overexpressing MITF, transcription factor EB or TFE3 are able to reorganize the lysosomal pathway and inhibit cellular senescence. The cell cycle analysis performed in the present study demonstrated that TFE3 overexpression promoted DNA synthesis and accelerated the cell cycle progression from $\mathrm{G} 1$ to $\mathrm{S}$ phase. The results of the present study may help to explain how TFE3 overexpression promotes ACHN cell proliferation. Giangrande et al (15) identified the TFE3 transcription factor to be a specific partner for E2F3, which is able to rescue retinoblastoma associated protein $(\mathrm{Rb})$-mediated growth arrest, providing a functional link between TFE3 and the Rb/E2F pathway. Leone et al (16) proposed that E2F3 activity serves an important role in the cell cycle of proliferating cells, controlling the expression of genes whose products limit the initiation of DNA replication, imparting a more marked control of $S$ phase than may otherwise be achieved by post-transcriptional regulation alone. The results of the present study demonstrated that TFE3 overexpression likely promoted ACHN cell proliferation through cell cycle regulation, which is consistent with previous studies.

Transcription factors and cell signaling pathways serve important roles in carcinogenesis $(17,18)$. The activation of membrane growth factor receptors drives cell proliferation signals through at least two biochemical pathways: the 
PI3K/AKT/mTOR and Ras/MAPK pathway. Overt activation of the PI3K/Akt/mTOR pathway has been observed in various types of human cancer, including RCCs (19). Previous investigations have demonstrated that rpS6, the best-characterized downstream effector of mTOR complex 1 , is upregulated in primary renal tumors with metastasis $(19,20)$. Immunoreactivity for rpS6 may be used as a measure of the activation of the mTOR pathway, which promotes cell growth. In addition, rpS6 may be an important tool for stratifying patients with metastatic RCC into different risk groups and improving patient selection for mTOR-targeted therapies (21). Argani et al (22) demonstrated that targeting the mTOR signaling pathway may be an effective way to treat Xp11.2 translocation RCCs. The present study demonstrated that the mTOR and p-rpS6 levels were increased in the OE group compared with the NC group, suggesting that TFE3 overexpression improved the proliferative capacity of ACHN cells through the activation of the mTOR pathway.

In conclusion, the results of the present study indicated that an increase in TFE3 expression resulted in malignant phenotypes in renal adenocarcinoma ACHN cells. TFE3 may serve an important role in the regulation of the cell cycle and cell proliferation. Additionally, the results suggested that mTOR and rpS6 may be effective therapeutic targets for Xp11.2 translocation RCCs.

\section{Acknowledgements}

The present study was supported by the National Natural Science Foundation of China (grant no. 81372743, to X.J.Z.).

\section{References}

1. Ramphal R, Pappo A, Zielenska M, Grant R and Ngan BY: Pediatric renal cell carcinoma: Clinical, pathologic, and molecular abnormalities associated with the members of the mit transcription factor family. Am J Clin Pathol 126: 349-364, 2006.

2. Komai Y, Fujiwara M, Fujii Y, Mukai H, Yonese J, Kawakami S, Yamamoto S, Migita T, Ishikawa Y, Kurata M, et al: Adult Xp11 translocation renal cell carcinoma diagnosed by cytogenetics and immunohistochemistry. Clin Cancer Res 15: 1170-1176, 2009.

3. Lopez-Beltran A, Cheng L, Vidal A, Scarpelli M, Kirkali Z, Blanca A and Montironi R: Pathology of renal cell carcinoma: An update. Anal Quant Cytopathol Histpathol 35: 61-76, 2013.

4. Argani P, Lal P, Hutchinson B, Lui MY, Reuter VE and Ladanyi M: Aberrant nuclear immunoreactivity for TFE3 in neoplasms with TFE3 gene fusions: A sensitive and specific immunohistochemical assay. Am J Surg Pathol 27: 750-761, 2003.

5. Argani P, Antonescu CR, Illei PB, Lui MY, Timmons CF, Newbury R, Reuter VE, Garvin AJ, Perez-Atayde AR, Fletcher JA, et al: Primary renal neoplasms with the ASPL-TFE3 gene fusion of alveolar soft part sarcoma: A distinctive tumor entity previously included among renal cell carcinomas of children and adolescents. Am J Pathol 159: 179-192, 2001.

6. Argani P, Olgac S, Tickoo SK, Goldfischer M, Moch H, Chan DY, Eble JN, Bonsib SM, Jimeno M, Lloreta J, et al: Xp11 translocation renal cell carcinoma in adults: Expanded clinical, pathologic, and genetic spectrum. Am J Surg Pathol 31: 1149-1160, 2007.
7. Castellvi J, Garcia A, Ruiz-Marcellan C, Hernández-Losa J, Peg V, Salcedo M, Gil-Moreno A, Ramon y and Cajal S: Cell signaling in endometrial carcinoma: Phosphorylated 4E-binding protein-1 expression in endometrial cancer correlates with aggressive tumors and prognosis. Hum Pathol 40: 1418-1426, 2009.

8. Parikh J, Coleman T, Messias N and Brown J: Temsirolimus in the treatment of renal cell carcinoma associated with Xp11.2 translocation/TFE gene fusion proteins: A case report and review of literature. Rare Tumors 1: e53, 2009.

9. Choueiri TK, Lim ZD, Hirsch MS, Tamboli P, Jonasch E, McDermott DF, Dal Cin P, Corn P, Vaishampayan U, Heng DY and Tannir NM: Vascular endothelial growth factor-targeted therapy for the treatment of adult metastatic Xp11.2 translocation renal cell carcinoma. Cancer 116: 5219-5225, 2010.

10. Rao Q, Williamson SR, Zhang S, Eble JN, Grignon DJ, Wang M, Zhou XJ, Huang W, Tan PH, Maclennan GT and Cheng L: TFE3 break-apart FISH has a higher sensitivity for Xp11.2 translocation-associated renal cell carcinoma compared with TFE3 or cathepsin $\mathrm{K}$ immunohistochemical staining alone: Expanding the morphologic spectrum. Am J Surg Pathol 37: 804-815, 2013.

11. Dickson BC, Brooks JS, Pasha TL and Zhang PJ: TFE3 expression in tumors of the microphthalmia-associated transcription factor (MiTF) family. Int J Surg Pathol 19: 26-30, 2011.

12. Rao Q, Guan B and Zhou XJ: Xp11.2 Translocation renal cell carcinomas have a poorer prognosis than non-Xp11.2 translocation carcinomas in children and young adults: A meta-analysis. Int J Surg Pathol 18: 458-464, 2010.

13. Xu L, Yang R, Gan W, Chen X, Qiu X, Fu K, Huang J, Zhu G and Guo H: Xp11.2 translocation renal cell carcinomas in young adults. BMC Urol 15: 57, 2015.

14. Ploper D and De Robertis EM: The MITF family of transcription factors: Role in endolysosomal biogenesis, Wnt signaling, and oncogenesis. Pharmacol Res 99: 36-43, 2015.

15. Giangrande PH, Hallstrom TC, Tunyaplin C, Calame K and Nevins JR: Identification of E-Box factor TFE3 as a functional partner for the E2F3 transcription factor. Mol Cell Biol 23: 3707-3720, 2003.

16. Leone G, Degregori J, Yan Z, Jakoi L, Ishida S, Williams RS and Nevins JR: E2F3 activity is regulated during the cell cycle and is required for the induction of S phase. Genes Dev 12: 2120-2130, 1998.

17. Li M, Wang $\mathrm{Y}, \mathrm{Yu} \mathrm{Y}$, Nishizawa $\mathrm{M}$, Nakajima T, Ito $\mathrm{S}$ and Kannan P: The human transcription factor activation protein-2 gamma (AP-2gamma): Gene structure, promoter and, expression in mammary carcinoma cell lines. Gene 301: 43-51, 2002.

18. Yu Y, Wang Y, Li M and Kannan P: Tumorigenic effect of transcription factor hAP-2alpha and the intricate link between hAP-2alpha activation and squelching. Mol Carcinog 34: 172-179, 2002.

19. Furuya N, Kamai T, Shirataki H, Yanai Y, Fukuda T, Mizuno T, Nakamura F, Kambara T, Nakanishi K, Abe H and Yoshida K: Serum interferon alpha receptor 2 mRNA may predict efficacy of interferon alpha with/without low-dose sorafenib for metastatic clear cell renal cell carcinoma. Cancer Immunol Immunother 60: 793-808, 2011

20. Kamai T, Tsujii T, Arai K, Takagi K, Asami H, Ito $\mathrm{Y}$ and Oshima H: Significant association of Rho/ROCK pathway with invasion and metastasis of bladder cancer. Clin Cancer Res 9: 2632-2641, 2003

21. Pantuck AJ, Seligson DB, Klatte T, Yu H, Leppert JT, Moore L, O'Toole T, Gibbons J, Belldegrun AS and Figlin RA: Prognostic relevance of the mTOR pathway in renal cell carcinoma: Implications for molecular patient selection for targeted therapy. Cancer 109: 2257-2267, 2007.

22. Argani P, Hicks J, De Marzo AM, Albadine R, Illei PB, Ladanyi M, Reuter VE and Netto GJ: Xp11 translocation renal cell carcinoma (RCC): Extended immunohistochemical profile emphasizing novel RCC Markers. Am J Surg Pathol 34: 1295-1303, 2010. 